\title{
Clinico-pathological relationship between androgen receptor and tumour infiltrating lymphocytes in triple negative breast cancer
}

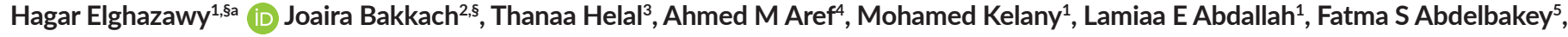 \\ Dalia Ali ${ }^{1}$, Doaa Z Ali ${ }^{4}$, Mai O Ahmed ${ }^{4}$, Amer Ali Abd El-Hafeez ${ }^{6,7}$, Pradipta Ghosh ${ }^{7,8,9,10}$ and Mohamed O Alorabi ${ }^{1}$ \\ ${ }^{1}$ Department of Clinical Oncology, Faculty of Medicine, Ain Shams University, Cairo, 11591, Egypt \\ ${ }^{2}$ Biomedical Genomics \& Oncogenetics Research Laboratory, Faculty of Sciences and Techniques of Tangier, Abdelmalek Essaadi University, Tangier, \\ 90 000, Morocco \\ ${ }^{3}$ Department of Pathology, Faculty of Medicine, Ain Shams University, Cairo, 11591, Egypt \\ ${ }^{4}$ Faculty of Biotechnology, October University for Modern Sciences and Arts (MSA), Giza, 12451, Egypt \\ ${ }^{5}$ Department of Clinical Oncology, Electricity Hospital, Cairo, 11775, Egypt \\ ${ }^{6}$ Pharmacology and Experimental Oncology Unit, Cancer Biology Department, National Cancer Institute, Cairo University, Cairo, 11796, Egypt \\ 'Department of Cellular and Molecular Medicine, School of Medicine, University of California, San Diego, La Jolla, CA 92093, USA \\ ${ }^{8}$ Department of Medicine, University of California, San Diego, La Jolla, CA 92093, USA \\ ${ }^{9}$ Rebecca and John Moore Comprehensive Cancer Center, University of California, San Diego, La Jolla, CA 92037, USA \\ ${ }^{10}$ Veterans Affairs Medical Center, La Jolla, CA 92161, USA \\ ${ }^{\S}$ Hagar Elghazawy and Joaira Bakkach had contributed equally to the work \\ ahttps://orcid.org/0000-0001-6839-4147
}

\section{Abstract}

Background: Triple negative breast cancer (TNBC) is an aggressive subtype of breast cancer (BC) with ill-defined therapeutic targets. Androgen receptor (AR) and tumour-infiltrating lymphocytes (TILs) had a prognostic and predictive value in TNBC. The relationship between AR, TILs and clinical behaviour is still not fully understood.

Methods: Thirty-six TNBC patients were evaluated for AR (positive if $\geq 1 \%$ expression), CD3, CD4, CD8 and CD20 by immunohistochemistry. Stromal TILs were quantified following TILs Working Group recommendations. Lymphocyte-predominant breast cancer (LPBC) was defined as stromal TILs $\geq 50 \%$, whereas lymphocyte-deficient breast cancer (LDBC) was defined as $<50 \%$.

Results: The mean age was 52.5 years and $27.8 \%$ were $\geq 60$ years. Seven patients $(21.2 \%)$ were AR+. All AR+ cases were postmenopausal ( $\geq 50$ years old). LPBC was $32.2 \%$ of the whole cohort. Median TILs were $37.5 \%$ and $10 \%(p=0.1)$ and median CD20 was $20 \%$ and $7.5 \%(p=0.008)$ in AR- and AR+, respectively. Mean CD3 was $80.7 \%$ and $93.3 \%$ ( $p=0.007)$ and CD8 was $75 \%$ and $80.8 \%(p=0.41)$ in AR- and AR+, respectively. All patients who were $\geq 60$ years old expressed CD20. LDBC was found to be significantly higher in $\mathrm{N}+$ versus $\mathrm{N}$ - patients $(p=0.03$ ) with median TILs of $20 \%$ versus $50 \%$ in $\mathrm{N}+$ versus $\mathrm{N}$-, respectively $(p=0.03$ ). LDBC was associated with higher risk of lymph node $(\mathrm{LN}$ ) involvement (odds ratio $=6 ; 95 \% \mathrm{Cl}=1.05-34.21 ; p=0.04$ ).

Conclusions: AR expression was evident in older age ( $\geq 50$ years). Median CD20 was higher in AR- TNBC, while mean CD3 was higher in AR+ tumours. LDBC was associated with higher risk of $L N$ involvement. Larger studies are needed to focus on the clinical impact of the relation between AR and TILs in TNBC.

Correspondence to: Hagar Elghazawy Email: dr.hagar.elghazawy@med.asu.edu.eg

ecancer 2021, 15:1317

https://doi.org/10.3332/ecancer.2021.1317

Published: $11 / 11 / 2021$

Received: 08/07/2021

Publication costs for this article were supported by ecancer (UK Charity number 1176307).

Copyright: (c) the authors; licensee ecancermedicalscience. This is an Open Access article distributed under the terms of the Creative Commons Attribution License (http:// creativecommons.org/licenses/by/4.0), which permits unrestricted use, distribution, and reproduction in any medium, provided the original work is properly cited. 
Keywords: triple negative breast cancer, TNBC, androgen receptor, AR, tumour infiltrating lymphocytes, TILs

\section{Background}

Triple negative breast cancer (TNBC) is a challenging heterogeneous disease with distinct molecular subtypes that does not have receptors for oestrogen, progesterone hormones and the human epidermal growth factor receptor 2 (HER2) protein. TNBC was grouped into six molecular subtypes: basal-like (BL) 1, BL2, mesenchymal (M), mesenchymal stem-like (MSL), immunomodulatory (IM) and luminal androgen receptor (LAR) [1]. But thereafter, Lehmann et al [2] found that transcripts in the previously defined IM and MSL subtypes came from tumour-infiltrating lymphocytes (TILs) and tumour-associated stromal cells, respectively, and they reduced the number of TNBC molecular subtypes to four (BL1, BL2, M and LAR).

TILs play an essential role in predicting response to chemotherapy and improving clinical outcomes in breast cancer (BC). Moreover, as the immunotherapy landscape continues to evolve, there is interest in whether the immune system could be playing a more substantial role in TNBC specifically. The association between TNBC subtypes and the impact of TILs is still not fully understood. However, accumulating evidence from several studies indicates that intra-tumoural levels of TILs in TNBC are: a) predictive for response to neo-adjuvant chemotherapy and b) prognostic in patients treated with adjuvant chemotherapy, being correlated with improved overall survival (OS) and disease free survival (DFS) [3].

Besides the immune cell markers, the androgen receptor (AR), which controls the transcription of different genes including the immune response genes, has been recognised as a valuable biomarker in TNBC [4]. The AR expression was correlated with better survival outcomes in TNBC [5], albeit its clinical utility and immunological impact remain unclear. However, many opened questions still need to be answered such as what is the prevalence of AR positivity in TNBC and whether AR expression correlates with the mean TILs or with CD3, CD4, CD8, CD20 expression. Also, it remains not fully clear whether there is any relationship between the predominance of TILs and the age or stage. Here, we addressed these questions and explored the correlation between the AR expression and the total and differential TILs in TNBC.

\section{Methods}

In this cross-sectional, pilot study, patients' records were reviewed retrospectively to select patients with TNBC. TNBC was defined based on the American Society of Clinical Oncology/College of American Pathologists (ASCO/CAP) recommendations (2010) [6], as tumours with negative ( $<1 \%$ of nuclear staining) oestrogen receptor (ER), progesterone receptor (PR) and lack HER2 receptor overexpression or oncogene amplification. From a cohort of 800 BC patients who were diagnosed in 2012, at the clinical oncology department, Ain Shams University; $10 \%$ (80 patients) were diagnosed as TNBC in this year; of whom 36 patients had available tumour paraffin tissue and medical records. The clinico-pathological data and survival outcomes were collected. Tumour (T), nodes (N) and metastases (M) (TNM) staging was done according to the seventh edition of the American Joint Committee on Cancer (AJCC). The study protocol was approved by the Research Ethics committee, Faculty of Medicine, Ain Shams University, Cairo, Egypt.

Pathological evaluation was performed by a dedicated pathologist (TH), who was blinded for the clinical data. Haematoxylin and eosinstained sections were revised for the negativity of ER, PR and Her2 and assessed for the histologic type and grade of the tumour. Then, the sections were examined to quantify the stromal TILs according to the 2014 TILs International Working Group [7], where it was defined as the percentage of lymphocytes in direct contact with tumour cells. Lymphocyte-predominant breast cancer (LPBC) was defined as TILs $\geq 50 \%$, while lymphocytic deficient breast cancer (LDBC) was defined as TILs $<50 \%$.

Formalin-fixed, paraffin-embedded tissue specimens were available for the evaluation of both of AR and TILs in 28 patients, AR alone in 5 patients and TILs alone in 3 patients. The AR expression (Code 200M-18) was evaluated by immunohistochemistry (IHC), 
and considered positive if $\geq 1 \%$ nuclear staining of the tumour cells [4]. Also, immunostaining was performed for $T$ cell markers CD3 (Code 00000 51564), CD4 (Code 104R-28), CD8 (Code 108M-98) and B cell marker CD20 (Code 00000 27500). All antibodies were ready to use, from Cell Marque, California, USA. CD3, CD4, CD8 and CD20 immunostaining results were evaluated as mean percentage of the stained lymphocytes in relation to the total lymphocytes in the whole tissue section. Then the mean (for CD3 and CD8) and median (for CD4 and CD20) were calculated. The primary aim of our study was to describe the expression of AR and immune cells (CD3, CD4, CD8 and CD20) in TNBC, and the percentage of TILs as well. The secondary aim was to correlate the clinico-pathological parameters with these biomarkers.

\section{Statistical analysis}

Recorded data were analysed using the Statistical Package for Social Sciences, version 20.0 (SPSS Inc., Chicago, Illinois, USA). Quantitative data were expressed as mean \pm standard deviation (SD) or median and interquartile range (IQR). Qualitative data were expressed as frequency and percentage. Independent-samples $t$-test of significance and Mann-Whitney ( $z$ ) test were used to compare two means and non-parametric data, respectively. Analysis of Variance (ANOVA) test and Kruskal-Wallis test were used to compare more than two means and multiple-group comparisons in non-parametric data, respectively. Chi-square $\left(x^{2}\right)$ test was used in order to compare proportions between qualitative parameters. As multivariate analysis is not suitable for small sets of data, estimates are represented according to univariate analysis. Spearman's correlation coefficient $(r)$ test was used to assess the degree of association between two sets of variables. The $p$-value was considered significant if $\leq 0.05$.

\section{Results}

\section{Patient characteristics}

Thirty-six TNBC patients with available enough tumour material were identified for analysis. The patients' characteristics are shown in Supplementary Material, Table S1. The mean age at diagnosis was 52.5 years (range: $30-75$ years), and $27.8 \%$ of cases were $\geq 60$ years old. Most of the tumours (58.3\%) were of invasive duct carcinoma (IDC) type, while medullary carcinoma and invasive lobular carcinoma (ILC) accounted for $22 \%$ and $11 \%$, respectively. Grade II and III tumours were $30.6 \%$ and $52.8 \%$, respectively. Stages I, II, III and IV represented $5.6 \%, 30.5 \%, 52.7 \%$ and $8.3 \%$, respectively. Lymph nodes (LNs) were positive in $77.8 \%$ (28 patients). After a median follow-up of 39 months, nine patients had developed a disease progression and the 3-year OS was reached in $44.4 \%$ of the patients.

\section{AR expression and its relation with the clinico-pathological and survival parameters}

AR was tested in 33 patients and it was expressed in $21.2 \%$ (7 patients). All AR+ cases (100\%) were postmenopausal ( $\geq 50$ years old). Although patients with AR+ tumours were older than those who were AR- (mean age: 55 versus 51.6 years), there was no statistically significant difference in age between the two groups $(p=0.47)$. LNs were involved in $77 \%$ and $85.7 \%$ in AR- and AR+, respectively, $(p=0.61)$. No statistical difference was found in median OS between AR- and AR+ groups (31.5 versus 25 months, $p=0.77$ ). The clinico-pathological parameters according to AR expression are shown in Table 1.

The majority of AR+ tumours (85.7\%) was LDBC subtype, with median percentage of TILs was $37.5 \%$ and $10 \%$ in AR- and AR+ tumours, respectively, ( $p=0.10)$. Median CD20 was significantly higher in AR-versus AR+ (20\% versus $7.5 \%$, respectively, $p=0.008)$, as depicted in Figure1a, while mean CD3 was significantly lower in AR- versus AR+ (80.7\% versus 93.3\%, respectively, $p=0.007)$, as depicted in Figure 1b. On the other side, median CD4 and mean CD8 were not statistically different between AR- and AR+ tumours. Table 2 illustrated the correlation between the AR and total \& differential TILs expression. 
Table 1. The clinico-pathological parameters according to AR expression.

\begin{tabular}{|c|c|c|c|c|c|c|c|}
\hline \multirow{2}{*}{\multicolumn{2}{|c|}{ Clinico-pathological parameters }} & \multicolumn{2}{|c|}{$A R-(26)$} & \multicolumn{2}{|c|}{$\mathrm{AR}+(7)$} & \multirow{3}{*}{$\begin{array}{c}\begin{array}{c}\text { Chi-square } \\
\text { test }\end{array} \\
-0.732^{\mathrm{a}}\end{array}$} & \multirow{3}{*}{$\begin{array}{c}p \text {-value } \\
0.470\end{array}$} \\
\hline & & \multirow{2}{*}{$\begin{array}{c}\text { No. } \\
51.65 \pm 11.76 \\
30-75\end{array}$} & \multirow{2}{*}{$\%$} & \multirow{2}{*}{$\begin{array}{c}\text { No. } \\
55.00 \pm 4.40 \\
50-63\end{array}$} & \multirow{2}{*}{$\%$} & & \\
\hline Age & $\begin{array}{l}\text { Mean } \pm \text { SD } \\
\text { Range }\end{array}$ & & & & & & \\
\hline Age category & $\begin{array}{l}<60 \text { years } \\
\geq 60 \text { years }\end{array}$ & $\begin{array}{c}18 \\
8\end{array}$ & $\begin{array}{l}69.2 \\
30.8\end{array}$ & $\begin{array}{l}6 \\
1\end{array}$ & $\begin{array}{l}85.7 \\
14.3\end{array}$ & 0.755 & 0.385 \\
\hline \multirow{2}{*}{ Menopausal status } & Pre-menopausal & 9 & 34.6 & 0 & 0.0 & \multirow{2}{*}{3.332} & \multirow{2}{*}{0.068} \\
\hline & Post-menopausal & 17 & 65.4 & 7 & 100 & & \\
\hline Laterality & $\begin{array}{l}\text { Right } \\
\text { Left } \\
\text { Bilateral } \\
\text { Unknown }\end{array}$ & $\begin{array}{c}12 \\
12 \\
1 \\
1\end{array}$ & $\begin{array}{c}46.2 \\
46.2 \\
3.8 \\
3.8\end{array}$ & $\begin{array}{l}4 \\
3 \\
0 \\
0\end{array}$ & $\begin{array}{c}57.1 \\
42.9 \\
0.0 \\
0.0\end{array}$ & 0.689 & 0.876 \\
\hline Pathology & $\begin{array}{l}\text { IDC } \\
\text { Medullary carcinoma } \\
\text { ILC } \\
\text { Adenoid cystic } \\
\text { Unknown } \\
\end{array}$ & $\begin{array}{c}14 \\
7 \\
3 \\
1 \\
1 \\
\end{array}$ & $\begin{array}{c}53.9 \\
26.9 \\
11.6 \\
3.8 \\
3.8 \\
\end{array}$ & $\begin{array}{l}5 \\
1 \\
1 \\
0 \\
0\end{array}$ & $\begin{array}{c}71.4 \\
14.3 \\
14.3 \\
0.0 \\
0.0\end{array}$ & 3.476 & 0.627 \\
\hline \multirow{3}{*}{ Grade } & Grade II & 9 & 34.6 & 2 & 28.6 & \multirow{3}{*}{2.640} & \multirow{3}{*}{0.267} \\
\hline & Grade III & 11 & 42.3 & 5 & 71.4 & & \\
\hline & Unknown & 6 & 23.1 & 0 & 0.0 & & \\
\hline TNM staging & $\begin{array}{l}\text { I } \\
\text { II } \\
\text { III } \\
\text { IV } \\
\text { Unknown } \\
\end{array}$ & $\begin{array}{c}2 \\
7 \\
15 \\
1 \\
1 \\
\end{array}$ & $\begin{array}{c}7.7 \\
26.9 \\
57.8 \\
3.8 \\
3.8 \\
\end{array}$ & $\begin{array}{l}0 \\
3 \\
4 \\
0 \\
0\end{array}$ & $\begin{array}{c}0.0 \\
42.9 \\
57.1 \\
0.0 \\
0.0\end{array}$ & 1.539 & 0.820 \\
\hline T stage & $\begin{array}{l}\text { T1 } \\
\text { T2 } \\
\text { T3 } \\
\text { T4 } \\
\text { Unknown } \\
\end{array}$ & $\begin{array}{c}5 \\
10 \\
6 \\
4 \\
1\end{array}$ & $\begin{array}{c}19.2 \\
38.5 \\
23.1 \\
15.4 \\
3.8\end{array}$ & $\begin{array}{l}0 \\
5 \\
1 \\
1 \\
0\end{array}$ & $\begin{array}{c}0.0 \\
71.4 \\
14.3 \\
14.3 \\
0.0\end{array}$ & 3.139 & 0.535 \\
\hline LN category & $\begin{array}{l}\mathrm{NO} \\
\mathrm{N}+\end{array}$ & $\begin{array}{c}6 \\
20\end{array}$ & $\begin{array}{l}23.1 \\
76.9\end{array}$ & $\begin{array}{l}1 \\
6\end{array}$ & $\begin{array}{l}14.3 \\
85.7\end{array}$ & 0.255 & 0.614 \\
\hline Relapse/progression & $\begin{array}{l}\text { Negative } \\
\text { Positive } \\
\text { Unknown }\end{array}$ & $\begin{array}{c}19 \\
7 \\
0\end{array}$ & $\begin{array}{c}73.1 \\
26.9 \\
0.0 \\
\end{array}$ & $\begin{array}{l}6 \\
1 \\
0\end{array}$ & $\begin{array}{c}85.7 \\
14.3 \\
0.0 \\
\end{array}$ & 0.480 & 0.489 \\
\hline Median OS & $\begin{array}{l}\text { Median (IQR) } \\
\text { Range }\end{array}$ & $\begin{array}{c}31.5(18-44) \\
1.5-216\end{array}$ & & $\begin{array}{c}25(17-77) \\
4-86 \\
\end{array}$ & & $-0.286^{b}$ & 0.775 \\
\hline 3-year OS & $\begin{array}{l}<3 \text { years } \\
\geq 3 \text { years }\end{array}$ & $\begin{array}{l}14 \\
12\end{array}$ & $\begin{array}{l}53.8 \\
46.2\end{array}$ & $\begin{array}{l}4 \\
3\end{array}$ & $\begin{array}{l}57.1 \\
42.9\end{array}$ & 0.024 & 0.876 \\
\hline
\end{tabular}

IDC, Invasive ductal carcinoma; ILC, Invasive lobular carcinoma; LN, Lymph node; IQR, Interquartile range; OS, Overall survival andependent $t$-test

'Mann-Whitney test 


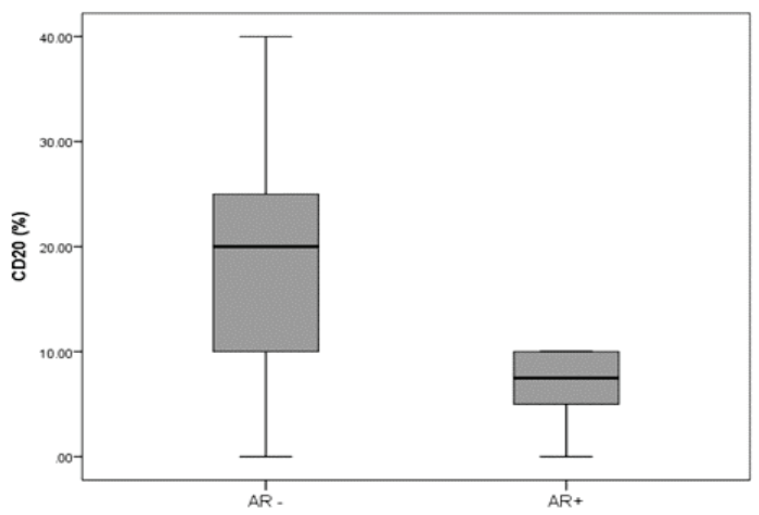

(a)

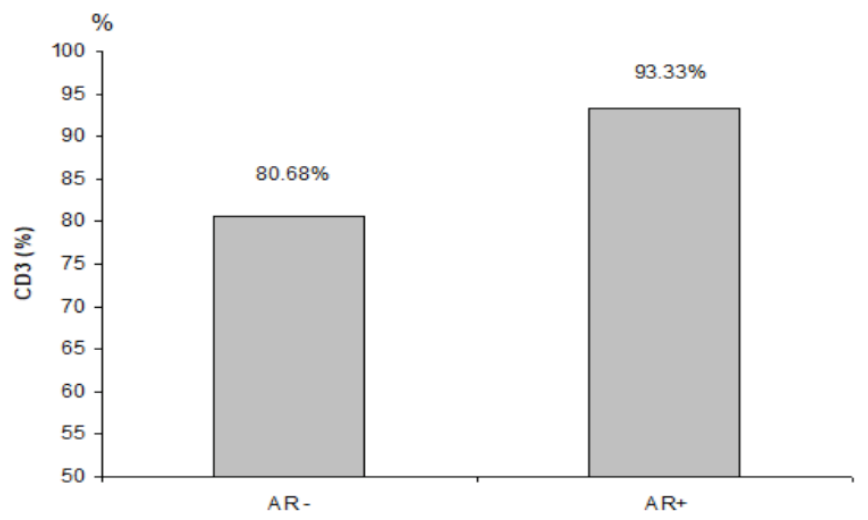

(b)

Figure 1. (a): Relation between AR and median CD20 expression ( $p=0.008)$. (b): Relation between AR and mean CD3 expression ( $p=0.007)$.

Table 2. The correlation between AR and total \& differential TILs expression. The $p$-value was considered significant if $\leq 0.05$.

\begin{tabular}{|c|c|c|c|c|c|}
\hline \multicolumn{2}{|c|}{ Variables } & $A R-(26)$ & $A R+(7)$ & Test value & $p$-value \\
\hline \multirow{2}{*}{ Median TILs (\%) } & Median (IQR) & $37.5(10-50)$ & $10(5-20)$ & \multirow{2}{*}{$-1.607^{c}$} & \multirow{2}{*}{0.108} \\
\hline & Range & $1-70$ & $3-40$ & & \\
\hline \multirow{3}{*}{ LPBC versus $\mathrm{LDBC}$} & LDBC & 14 (53.8\%) & $6(85.7 \%)$ & \multirow{3}{*}{$3.082^{\mathrm{a}}$} & \multirow{3}{*}{0.214} \\
\hline & LPBC & $8(30.8 \%)$ & $0(0.0 \%)$ & & \\
\hline & Unknown & $4(15.4 \%)$ & $1(14.3 \%)$ & & \\
\hline \multirow{2}{*}{ Median CD20 (\%) } & Median (IQR) & $20(10-25)$ & $7.5(5-10)$ & \multirow{2}{*}{$-2.643^{c}$} & \multirow{2}{*}{0.008} \\
\hline & Range & $0-40$ & $0-10$ & & \\
\hline \multirow{3}{*}{ CD20 expression } & Negative & $2(7.7 \%)$ & $1(14.3 \%)$ & \multirow{3}{*}{$0.290^{a}$} & \multirow{3}{*}{0.865} \\
\hline & Positive & $20(76.9 \%)$ & 5 (71.4\%) & & \\
\hline & Unknown & $4(15.4 \%)$ & $1(14.3 \%)$ & & \\
\hline \multirow{2}{*}{ Mean CD3 (\%) } & Mean \pm SD & $80.7 \pm 10.1$ & $93.3 \pm 4.1$ & \multirow{2}{*}{$-2.954^{b}$} & \multirow{2}{*}{0.007} \\
\hline & Range & $60-100$ & $90-100$ & & \\
\hline \multirow{3}{*}{ CD3 expression } & Negative & $0(0.0 \%)$ & $0(0.0 \%)$ & \multirow{3}{*}{$0.005^{\mathrm{a}}$} & \multirow{3}{*}{0.943} \\
\hline & Positive & 22 (84.6\%) & $6(85.7 \%)$ & & \\
\hline & Unknown & $4(15.4 \%)$ & $1(14.3 \%)$ & & \\
\hline \multirow{2}{*}{ Median CD4 (\%) } & Median (IQR) & $0(0-10)$ & $12.5(0-20)$ & \multirow{2}{*}{$-1.435^{c}$} & \multirow{2}{*}{0.151} \\
\hline & Range & $0-20$ & $0-30$ & & \\
\hline \multirow{3}{*}{ CD4 expression } & Negative & 14 (53.8\%) & $2(28.6 \%)$ & \multirow{3}{*}{$1.786^{a}$} & \multirow{3}{*}{0.409} \\
\hline & Positive & $8(30.8 \%)$ & $4(57.1 \%)$ & & \\
\hline & Unknown & $4(15.4 \%)$ & $1(14.3 \%)$ & & \\
\hline \multirow{2}{*}{ Mean CD8 (\%) } & Mean \pm SD & $75.0 \pm 16.3$ & $80.8 \pm 10.2$ & \multirow{2}{*}{$-0.825^{b}$} & \multirow{2}{*}{0.417} \\
\hline & Range & $40-100$ & 70-95 & & \\
\hline
\end{tabular}


Table 2. The correlation between AR and total \& differential TILs expression. The $p$-value was considered significant if $\leq 0.05$. (Continued)

\begin{tabular}{|l|l|c|c|c|c|}
\hline \multirow{3}{*}{ CD8 expression } & Negative & $0(0.0 \%)$ & $0(0.0 \%)$ & \multirow{3}{*}{$0.005^{a}$} & \multirow{2}{*}{0.943} \\
\cline { 2 - 4 } & Positive & $22(84.6 \%)$ & $6(85.7 \%)$ & \\
\cline { 2 - 4 } & Unknown & $4(15.4 \%)$ & $1(14.3 \%)$ & & \\
\hline
\end{tabular}

IQR, Interquartile range; LPBC, Lymphocytic predominance breast cancer; LDBC, Lymphocytic deficient breast cancer

${ }^{\mathrm{a} C h i-s q u a r e ~ t e s t ~}$

bIndependent $t$-test

cMann-Whitney test

\section{Total and differential TILs expression and its relation with the clinico-pathological parameters}

In the 31 patients, where TILs were evaluated, the median TILs were $30 \%$ (range $=1 \%-70 \%$ ), while LDBC and LPBC were $67.7 \%$ and $32.3 \%$, respectively. CD20 and CD4 were negative in $9.6 \%$ and $54.8 \%$, respectively. Table 3 showed descriptive analysis of the total and differential TILs expression. When correlating the lymphocytic predominance with the clinico-pathological parameters (shown in Table 4), LDBC type was found to be significantly higher in $\mathrm{N}+$ versus $\mathrm{N}$ - patients $(p=0.03$ ), as depicted in Figure 2 . Median TILs were $20 \%$ versus $50 \%$ in $\mathrm{N}+$ versus $\mathrm{N}-$, respectively, $(p=0.03)$ as illustrated in Table $5 \mathrm{a}$ and $\mathrm{b}$. Total TILs expression $<50 \%$ (LDBC) was associated with higher risk of LN involvement (odds ratio $(\mathrm{OR})=6 ; 95 \% \mathrm{Cl}=1.05-34.21 ; p=0.04)$.

Table 3. Descriptive analysis of the total and differential TILs expression.

\begin{tabular}{|c|c|c|}
\hline \multicolumn{2}{|c|}{ Variables } & No. $=31$ \\
\hline \multirow{2}{*}{ Median TILs (\%) } & Median (IQR) & $30(10-50)$ \\
\hline & Range & $1-70$ \\
\hline \multirow{2}{*}{ LDBC versus $L P B C$} & LDBC & $21(67.7 \%)$ \\
\hline & LPBC & 10 (32.3\%) \\
\hline \multirow{2}{*}{ Median CD20 (\%) } & Median (IQR) & $15(10-20)$ \\
\hline & Range & $0-40$ \\
\hline \multirow{2}{*}{ CD20 expression } & Negative & $3(9.6 \%)$ \\
\hline & Positive & 28 (90.4\%) \\
\hline \multirow{2}{*}{ Mean CD3 (\%) } & Mean \pm SD & $80.5 \pm 17.7$ \\
\hline & Range & $2-100$ \\
\hline \multirow{2}{*}{ CD3 expression } & Negative & $0(0.0 \%)$ \\
\hline & Positive & 31 (100\%) \\
\hline \multirow{2}{*}{ Median CD4 (\%) } & Median (IQR) & $0(0-15)$ \\
\hline & Range & $0-30$ \\
\hline \multirow{2}{*}{ CD4 expression } & Negative & $17(54.8 \%)$ \\
\hline & Positive & $14(45.2 \%)$ \\
\hline \multirow{2}{*}{ Mean CD8 (\%) } & Mean \pm SD & $73.4 \pm 19.7$ \\
\hline & Range & $2-100$ \\
\hline \multirow{2}{*}{ CD8 expression } & Negative & $0(0.0 \%)$ \\
\hline & Positive & $31(100 \%)$ \\
\hline
\end{tabular}

IQR, Interquartile range; SD, Standard deviation; LPBC, Lymphocytic predominance breast cancer; LDBC, Lymphocytic deficient breast cancer 
Table 4. Relation between lymphocytic predominance and clinico-pathological parameters. The $p$-value was considered significant if $\leq 0.05$.

\begin{tabular}{|c|c|c|c|c|c|c|c|}
\hline \multicolumn{2}{|c|}{ Variables } & \multicolumn{2}{|c|}{ LDBC (= 21) } & \multicolumn{2}{|c|}{ LPBC (=10) } & \multirow{3}{*}{$\begin{array}{c}\text { Chi-square test } \\
0.007\end{array}$} & \multirow{3}{*}{$\begin{array}{c}p \text {-value } \\
0.935\end{array}$} \\
\hline \multirow{2}{*}{ Age } & Age $<60$ & 15 & $71.4 \%$ & 7 & $70.0 \%$ & & \\
\hline & Age $\geq 60$ & 6 & $28.6 \%$ & 3 & $30.0 \%$ & & \\
\hline Morphology & $\begin{array}{l}\text { IDC } \\
\text { Medullary } \\
\text { ILC } \\
\text { Adenoid cystic }\end{array}$ & $\begin{array}{c}12 \\
5 \\
3 \\
1\end{array}$ & $\begin{array}{c}57.1 \% \\
23.8 \% \\
14.3 \% \\
4.8 \%\end{array}$ & $\begin{array}{l}6 \\
3 \\
1 \\
0\end{array}$ & $\begin{array}{l}60.0 \% \\
30.0 \% \\
10.0 \% \\
0.0 \%\end{array}$ & 0.873 & 0.928 \\
\hline TNM staging & $\begin{array}{l}\text { I } \\
\text { II } \\
\text { III } \\
\text { IV }\end{array}$ & $\begin{array}{c}2 \\
6 \\
11 \\
2\end{array}$ & $\begin{array}{c}9.5 \% \\
28.6 \% \\
52.4 \% \\
9.5 \%\end{array}$ & $\begin{array}{l}0 \\
5 \\
4 \\
1\end{array}$ & $\begin{array}{c}0.0 \% \\
50.0 \% \\
40.0 \% \\
10.0 \%\end{array}$ & 2.045 & 0.563 \\
\hline \multirow{4}{*}{$\mathrm{N}$ stage } & NO & 3 & $14.3 \%$ & 5 & $50.0 \%$ & \multirow{4}{*}{4.762} & \multirow{4}{*}{0.190} \\
\hline & N1 & 7 & $33.3 \%$ & 2 & $20.0 \%$ & & \\
\hline & N2 & 9 & $42.9 \%$ & 2 & $20.0 \%$ & & \\
\hline & N3 & 2 & $9.5 \%$ & 1 & $10.0 \%$ & & \\
\hline \multirow{2}{*}{ LN category } & $\mathrm{N}-$ & 3 & $14.3 \%$ & 5 & $50.0 \%$ & \multirow{2}{*}{4.513} & \multirow{2}{*}{0.034} \\
\hline & $\mathrm{N}+$ & 18 & $85.7 \%$ & 5 & $50.0 \%$ & & \\
\hline \multirow{2}{*}{ OS } & Median (IQR) & 32 & $(18-72)$ & 25 & (12-39) & \multirow{2}{*}{$-0.993^{a}$} & \multirow{2}{*}{0.320} \\
\hline & Range & $2-216$ & & $9-48$ & & & \\
\hline \multirow{2}{*}{ 3-year OS } & $<3$-year OS & 11 & $52.4 \%$ & 6 & $60.0 \%$ & \multirow{2}{*}{0.159} & \multirow{2}{*}{0.690} \\
\hline & $\geq 3$-year OS & 10 & $47.6 \%$ & 4 & $40.0 \%$ & & \\
\hline
\end{tabular}

IDC, Invasive duct carcinoma; ILC, Invasive lobular carcinoma; LN, Lymph node; IQR, Interquartile range; OS, Overall survival aMann-Whitney test

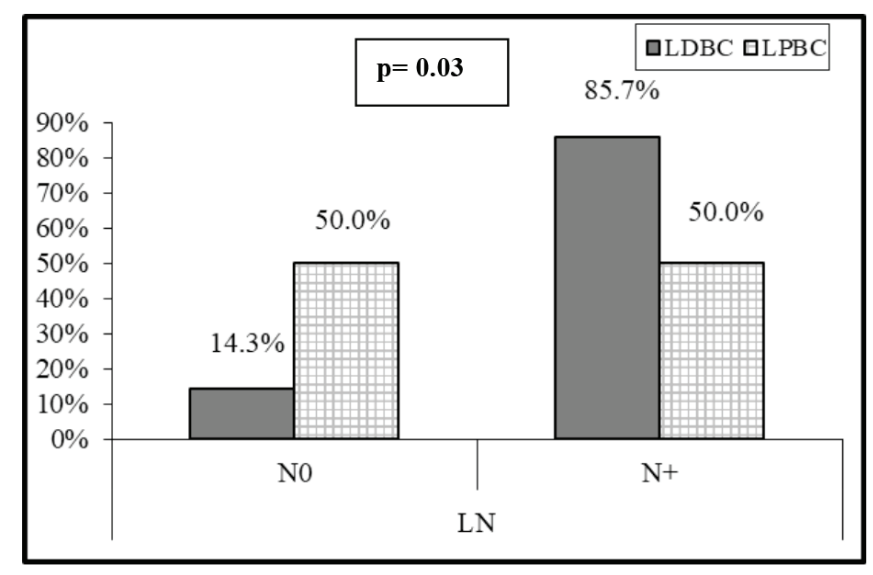

Figure 2. Relation between LN involvement and lymphocytic predominance.

On the other side, when analysing the relationship between the age and TILs (Supplementary Material, Table S2), it was found that median TILs were lower in patients $\geq 60$ years old despite statistically not significant, (median TILs $=10 \%$ versus $38 \%$ in $\geq 60$ years old versus $<60$ years old, respectively, $p=0.45$ ). Moreover, all patients who were $\geq 60$ years old expressed B-cell marker (100\%) 
(shown in Supplementary Material, Table S3). Furthermore, a significant positive correlation was present between CD8 and CD3 (correlation coefficient $(r)=0.591, p<0.001$ ), while significant inverse correlations were present between CD3 and CD20 $(r=-0.814$, $p<0.001)$, CD8 and CD20 ( $r=-0.382, p=0.03$ ) and CD8 and CD4 ( $r=-0.52, p=0.002)$, as illustrated in Figure 3a-c and Supplementary Material, Table S4.

Table 5A. Relation between LN involvement and the total and differential TILs. The $p$-value was considered significant if $\leq 0.05$.

\begin{tabular}{|c|c|c|c|c|c|}
\hline \multirow{2}{*}{\multicolumn{2}{|c|}{ Total and differential TILs }} & \multicolumn{2}{|c|}{ LN involvement } & \multirow{2}{*}{ Test value } & \multirow{2}{*}{$p$-value } \\
\hline & & \multirow{2}{*}{$\begin{array}{c}\mathrm{N}-(=8) \\
50(38-55)\end{array}$} & \multirow{2}{*}{$\frac{N+(=23)}{20(5-40)}$} & & \\
\hline \multirow{2}{*}{ Total TILs (\%) } & Median (IQR) & & & \multirow{2}{*}{$-2.159^{b}$} & \multirow{2}{*}{0.031} \\
\hline & Range & $10-60$ & $1-70$ & & \\
\hline \multirow{2}{*}{ Median CD20 (\%) } & Median (IQR) & $15(3-20)$ & $15(10-25)$ & \multirow{2}{*}{$-1.089^{b}$} & \multirow{2}{*}{0.276} \\
\hline & Range & $0-20$ & $0-40$ & & \\
\hline \multirow{2}{*}{ Mean CD3 (\%) } & Mean \pm SD & $85.6 \pm 9.8$ & $78.8 \pm 19.6$ & \multirow{2}{*}{$0.940^{a}$} & \multirow{2}{*}{0.355} \\
\hline & Range & $70-100$ & $2-100$ & & \\
\hline \multirow{2}{*}{ Median CD4 (\%) } & Median (IQR) & $5(0-13)$ & $0(0-20)$ & \multirow{2}{*}{$-0.074^{b}$} & \multirow{2}{*}{0.941} \\
\hline & Range & $0-20$ & $0-30$ & & \\
\hline \multirow{2}{*}{ Mean CD8 (\%) } & Mean \pm SD & $74.4 \pm 15.0$ & $73.1 \pm 21.4$ & \multirow{2}{*}{$0.151^{\mathrm{a}}$} & \multirow{2}{*}{0.881} \\
\hline & Range & $50-100$ & $2-100$ & & \\
\hline
\end{tabular}

LN, Lymph node; IQR, Interquartile range; SD, Standard deviation

andependent $t$-test

bMann-Whitney test

Table 5B. Relation between LN involvement and the total and differential TILs. The $p$-value was considered significant if $\leq 0.05$.

\begin{tabular}{|c|c|c|c|c|c|c|c|}
\hline \multirow{2}{*}{\multicolumn{2}{|c|}{ Total and differential TILs }} & \multicolumn{4}{|c|}{ LN involvement } & \multirow{3}{*}{$\begin{array}{c}\text { Chi-square } \\
\text { test }\end{array}$} & \multirow{3}{*}{$p$-value } \\
\hline & & \multicolumn{2}{|c|}{$\mathrm{N}-$} & \multicolumn{2}{|c|}{$\mathrm{N}+$} & & \\
\hline & & No. & $\%$ & No. & $\%$ & & \\
\hline \multirow{2}{*}{ LDBC versus $L P B C$} & LDBC & 3 & 37.5 & 18 & 78.3 & \multirow{2}{*}{4.513} & \multirow{2}{*}{0.034} \\
\hline & LPBC & 5 & 62.5 & 5 & 21.7 & & \\
\hline \multirow{2}{*}{ CD20 expression } & Negative & 2 & 25.0 & 1 & 4.3 & \multirow{2}{*}{2.896} & \multirow{2}{*}{0.089} \\
\hline & Positive & 6 & 75.0 & 22 & 95.7 & & \\
\hline \multirow{2}{*}{ CD3 expression } & Negative & 0 & 0.0 & 0 & 0.0 & \multirow{2}{*}{ NA } & \multirow{2}{*}{ NA } \\
\hline & Positive & 8 & 100 & 23 & 100.0 & & \\
\hline \multirow{2}{*}{ CD4 expression } & Negative & 4 & 50.0 & 13 & 56.5 & \multirow{2}{*}{0.102} & \multirow{2}{*}{0.750} \\
\hline & Positive & 4 & 50.0 & 10 & 43.5 & & \\
\hline \multirow{2}{*}{ CD8 expression } & Negative & 0 & 0.0 & 0 & 0.0 & \multirow{2}{*}{ NA } & \multirow{2}{*}{ NA } \\
\hline & Positive & 8 & 100 & 23 & 100 & & \\
\hline
\end{tabular}

NA, Not applicable 


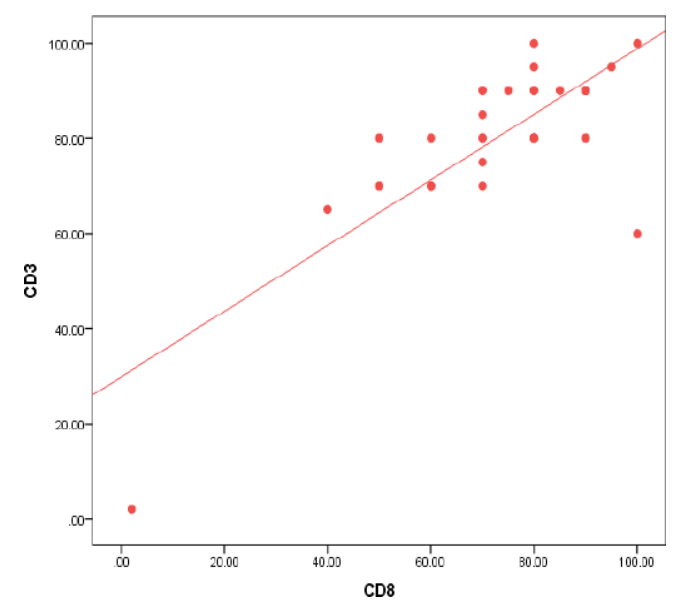

(a)

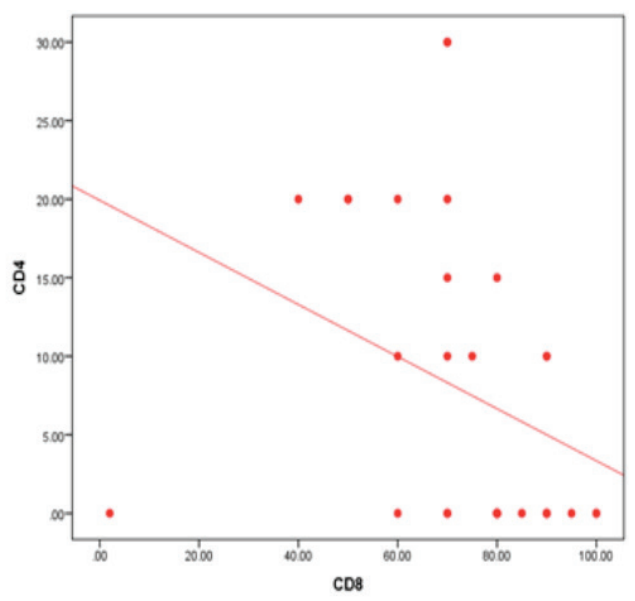

(b)

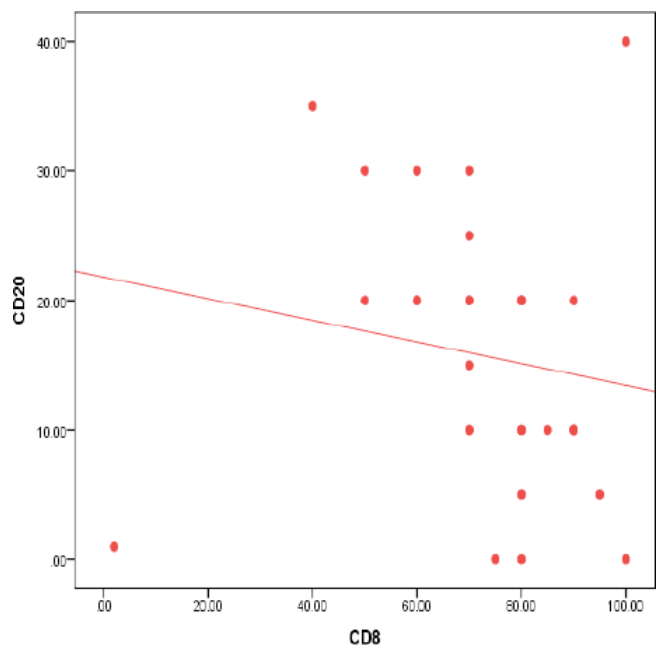

(c)

Figure 3. (a): Correlation between CD3 and CD8 expression (positive) ( $r=0.591, p \leq 0.001)$. (b): Correlation between CD4 and CD8 expression (inverse) $(r=-0.527, p=0.002)$. (c): Correlation between CD20 and CD8 expression (inverse) $(r=-0.382, p=0.034)$.

\section{Discussion}

It is well established that the expression of AR differs according to molecular subtypes of BC with more frequent expression in ER negative cancers. The prevalence of AR+ expressing tumours is generally ranging from $10 \%$ to $41 \%$ in TNBC cases [1, 8-14], with rare reports showing rates up to $79 \%[15,16]$. In accordance with most published reports, our rate of AR expression in TNBC was $21.2 \%$.

Whether clinico-pathologic characteristics of TNBC vary based on AR expression status have been extensively studied [8, 13, 15, 17, 18]. Some studies showed that patients with AR+ tumours were significantly older, exhibited tumours with significantly lower grades (I-II), more 
frequent nodal involvement, non-ductal histology and lower Ki67 [14, 15, 17, 18]. Other reports described reduced LN metastases in AR+ TNBCs [8], or just similar clinico-pathologic profile between AR+ and AR- TNBC tumours [13]. Herein, there was no statistically significant difference in the clinico-pathological parameters according to AR expression. However, AR+ cases were older in age and exhibited more regional nodal spread. Despite statistically insignificant, this profile was analogous to the LAR subtype described by Lehmann et al [2].

Available evidence about the prognostic value of AR in TNBC is controversial. Some reports suggested that AR-positivity was associated with good outcomes [8, 13], whereas others concluded that AR status conferred worse prognosis [19] or had no significant impact on disease prognosis [4, 20, 21]. Many factors may explain these discrepant results across studies including the sample size limited cohorts, differences in the ethnic origin, the anti-AR antibodies used for staining, staining/scoring method, as well as variability in the thresholds used to define AR positivity [4]. A meta-analysis published in 2017, demonstrated that AR-positive status was associated with better DFS and OS in TNBC (hazard ratio $(\mathrm{HR})=0.64 ; 95 \% \mathrm{Cl}=0.51-0.81 ; p<0.001$ and $\mathrm{HR}=0.64 ; 95 \% \mathrm{Cl}=0.49-0.88 ; p<0.001$, respectively), in univariate analysis [5]. Of note, no multivariate analysis was provided and this meta-analysis included heterogeneous studies in terms of methods of AR scoring, clinical cohorts' characteristics, therapies received and length of follow-up. A large multi-institutional study including about 1,407 TNBC tumours issued after this meta-analysis concluded that the AR-positivity was a marker of good prognosis in USA and Nigerian cohorts, whereas it conferred poor prognosis in Norway, Ireland and Indian cohorts, and was neutral in UK cohort [4]. Whereas a more recent meta-analysis (2020) [21] demonstrated that AR expression in TNBC was not associated with DFS $(\mathrm{HR}=0.92 ; 95 \% \mathrm{Cl}=0.67-1.27 ; p=$ 0.63), OS ( $\mathrm{HR}=0.91 ; 95 \% \mathrm{Cl}=0.67-1.22 ; p=0.53)$, distant-DFS ( $\mathrm{HR}=1.02 ; 95 \% \mathrm{Cl}=0.96-1.08 ; p=0.48)$ or recurrence-free survival (HR $=0.95 ; 95 \% \mathrm{Cl}=0.46-1.98 ; p=0.90)$, regardless of the confounding factors and heterogeneity that existed among included studies. Our study results had matched the latter meta-analysis results, where no statistical difference in median OS (31.5 versus 25 months, $p=0.77)$ or relapse/progression rate ( $26.9 \%$ versus $14.3 \%, p=0.48)$ was found between $A R-$ and $A R+$ groups.

Importantly, the presence of more frequent special histological subtypes with poor prognosis as medullary carcinoma, ILC and adenoid cystic carcinoma in the AR- versus the AR+ group (42\% versus $28 \%$ ) in our cohort, may have an impact on survival as pointed to by other studies [22].

Compared to other subtypes, TNBC was shown to exhibit higher levels of TILs [23]. There is heterogeneity of TILs cut-off used in published studies in order to distinguish between LPBC and LDBC. Some studies defined LPBC as showing more than 50\% of lymphocyte infiltration $[24,25]$, whereas others used different cut-offs [26]. In our cohort, median TILs were 30\% (range: 1\%-70\%), with a LPBC prevalence of $32.2 \%$, which is not in full agreement with other reports. Adams et al [24] reported much lower median TILs percentage (10\%), and with using the same cut-off of $\geq 50 \%$ TILs, only $4.4 \%$ were LPBC, whereas Pruneri et al [25] described a median TILs level of $20 \%$, with LPBC prevalence of $22 \%$ of cases.

Little is known about the association between TNBC clinico-pathologic features and lymphocytic predominance. A pooled analysis of nine large studies by Loi et al [26] demonstrated that TILs were significantly lower in older age. Whilst, Adams et al [24] reported no strong associations between TILs scores and age or menopausal status. Despite not statistically significant, we showed lower median TILs in patients $\geq 60$ years versus $<60$ years old $(10 \%$ versus $38 \%, p=0.45)$.

Interestingly, we found that patients with LN involvement were significantly more likely to be LDBC, where a total TILs expression < 50\% $(\mathrm{LDBC})$ was associated with higher risk of $\mathrm{LN}$ involvement $(\mathrm{OR}=6 ; 95 \% \mathrm{Cl}=1.05-34.21 ; p=0.04)$. This is in agreement with Loi et al [26], but in contrast to a recent meta-analysis which concluded that no significant association between decreased TILs and LN metastasis risk [27].

Our knowledge about the association between TILs and AR is still limited. In a large cohort study about non-metastatic TNBC of LAR subtype, this tumour subset was found to exhibit lower median stromal TILs and to be less likely LPBC ( $\geq 50 \%$ TILs) compared to non-LAR, although this did not reach statistical significance [28], similarly to our study. However, we did not examine the genetic profiles of our AR+ tumours to classify them into the LAR subtype. Other reports using IHC described significant association between AR expression and lower levels of stromal TILs $[11,17]$.

Studies about the immune cells subsets composition of TILs according to AR expression are very scarce. In our study, median CD20 was significantly higher in AR- tumours compared to those with AR+ (20\% versus $7.5 \%$, respectively, $p=0.008)$. Whereas, mean CD3 was significantly lower in AR- versus AR+ (80.7\% versus $93.3 \%$, respectively, $p=0.007)$. On the other side, previous publications reported that 
CD8+ were more frequent in AR+ than AR- tumours [12, 29, 30], in contrast to our study which showed that neither CD8 nor CD4 were statistically different between $A R+$ and $A R-$ tumours.

Based on two large-scale BC genomics data, evidence from a comprehensive analysis of 26 immune gene-sets including 15 immune cell type and function suggested that TNBC had the strongest tumour immunogenicity. Comparison of the immune infiltrate densities of different immune cell subpopulations demonstrated higher degree of infiltration in TNBC than non-TNBC, including CD3, CD8 and CD20 and others [31].

T-lymphocytes represent the main lymphocyte type in the tumour microenvironment, and the majority of T lymphocytes express a cytotoxic effector phenotype (CD8+). Intra-tumoural and adjacent stromal CD8+ T-cell infiltration have been found to be significantly associated with ER negativity and basal phenotype [32, 33]. Infiltrating CD8+ T-cells have been reported in more than 60\% of TNBC cases [33, 34]. In our study, CD8 was expressed in $100 \%$ of the cases with the mean of its expression was $73.4 \%$.

The role of tumour-infiltrating B cells (CD20) as components of TILs in BC subtypes is still unclear. A positive correlation between higher numbers of total CD20+ B cells and ER and PR negativity, and basal phenotype has been reported [35]. In our study, CD20 was expressed in $90.3 \%$ of the tumours and its median expression was significantly higher in AR- versus AR+ TNBC (20\% versus $7.5 \%$, respectively, $p=0.008)$.

Using a digital pathology computational workflow to quantify the spatial patterns of five immune markers (CD3, CD4, CD8, CD20 and FoxP3) in TNBC, Mi et al [36] demonstrated positive correlations between CD3 and CD8 cells. Similarly, we also showed a significant positive correlation between CD3 and CD8. Data from a study that used multiplexed ion beam imaging to simultaneously quantify in situ expression of 36 proteins in 41 patients with TNBC, suggested that all patients with B cells had also CD4 T cells and CD8 T cells [37]. In contrast, we found in our study significant inverse correlations between CD20 and CD8, as well as CD20 and CD3.

Immune cellular subpopulations in BC representing the innate immunity (natural killer, CD68+ and CD11c+ cells) and adaptive immunity (CD3+ cells (CD8+ or CD4+) and CD20+ cells) [38], worth thorough evaluation in TNBC, with the aim of understanding its clinical implications in BC management. In a recent consensus report for the management of TNBC, the majority of the panellists concluded that more evidence to support the predictive value of TILs and its impact on the clinical decision are warranted [39].

As no prior studies evaluating the exact relation between AR and TILs in this unique disease entity 'TNBC', we tended to represent all the statistical analyses and correlations which we evaluated, keeping with the aim of this exploratory study which may help future research. This study had mainly described the expression patterns of AR and TILs in TNBC. Moreover, the correlation between AR and the total and different TILs subpopulations was illustrated. TILs were evaluated by one pathologist who was blinded to the clinical characteristics and according to the International Working Group. However, our findings should be interpreted carefully. The limitations of our study include: i) the retrospective nature, ii) the small sample size (despite this, there were some significant correlations) and iii) the survival data was not mature due to the short follow-up duration (median: 39 months).

\section{Conclusions}

This study highlighted the probable relationship between the AR and total and differential TILs expression in TNBC; and the clinico-pathological characteristics as well. Understanding the immune micro-environment in a subset of tumours with poor prognosis and less identified therapeutic targets like TNBC, may pave the way for the advent of immunotherapy in specific group of patients. Moreover, lower TILs density may identify a subpopulation of TNBC who warrants more radical regional LNs management. The prognostic relevance and the potential predictive impact of AR and TILs in TNBCs merit further evaluation in larger scale studies.

\section{Conflicts of interest}

All authors declared no conflicts of interest. 


\section{Authors' contributions}

Conception and design: HE, JB, TH, AMA, MK, DZA, MO Ahmed, MO Alorabi

Acquisition and interpretation of data: all authors

Drafting the manuscript and revising it critically for important intellectual content: all authors.

\section{Funding}

None.

\section{References}

1. Lehmann B, Bauer J, and Chen X, et al (2011) Identification of human triple-negative breast cancer subtypes and preclinical models for selection of targeted therapies J Clin Invest 121(7) 2750-2767 https://doi.org/10.1172/JCI45014 PMID: 21633166 PMCID: 3127435

2. Lehmann B, Jovanović B, and Chen X, et al (2016) Refinement of triple-negative breast cancer molecular subtypes: implications for neoadjuvant chemotherapy selection. PLos One 11(6) e0157368 https://doi.org/10.1371/journal.pone.0157368 PMID: 27310713 PMCID: 4911051

3. Gao G, Wang Z, and Qu X, et al (2020) Prognostic value of tumor-infiltrating lymphocytes in patients with triple-negative breast cancer: a systematic review and meta-analysis BMC Cancer 20(1) 179 https://doi.org/10.1186/s12885-020-6668-z PMID: 32131780 PMCID: 7057662

4. Bhattarai S, Klimov S, and Mittal K, et al (2019) Prognostic role of androgen receptor in triple negative breast cancer: a multi-institutional study Cancers 11(7) 995 https://doi.org/10.3390/cancers11070995 PMCID: 6678933

5. Bozovic-Spasojevic I, Zardavas D, and Brohée S, et al (2016) The prognostic role of androgen receptor in patients with early-stage breast cancer: a meta-analysis of clinical and gene expression data Clin Cancer Res 23(11) 2702-2712 https://doi.org/10.1158/10780432.CCR-16-0979

6. Hammond ME, Hayes DF, and Dowsett M, et al (2010) American Society of Clinical Oncology/College of American Pathologists guideline recommendations for immunohistochemical testing of estrogen and progesterone receptors in breast cancer J Clin Oncol 28(16) 2784-95 https://doi.org/10.1200/JCO.2009.25.6529 PMID: 20404251 PMCID: 2881855

7. Salgado R, Denkert C, and Demaria S, et al (2015) The evaluation of tumor-infiltrating lymphocytes (TILs) in breast cancer: recommendations by an International TILs Working Group 2014 Ann Oncol 26(2) 259-271 https://doi.org/10.1093/annonc/mdu450

8. He J, Peng R, and Yuan Z, et al (2011) Prognostic value of androgen receptor expression in operable triple-negative breast cancer: a retrospective analysis based on a tissue microarray Med Oncol 29(2) 406-410 https://doi.org/10.1007/s12032-011-9832-0 PMID: 21264529

9. Astvatsaturyan K, Yue Y, and Walts A, et al (2018) Androgen receptor positive triple negative breast cancer: clinicopathologic, prognostic, and predictive features PLos One 13(6) e0197827 https://doi.org/10.1371/journal.pone.0197827 PMID: 29883487 PMCID: 5993259

10. Gucalp A, Tolaney S, and Isakoff S, et al (2013) Phase II trial of bicalutamide in patients with androgen receptor-positive, estrogen receptor-negative metastatic breast cancer Clin Cancer Res 19(19) 5505-5512 https://doi.org/10.1158/1078-0432.CCR-12-3327 PMID: 23965901 PMCID: 4086643

11. Leon-Ferre R, Polley M, and Liu H, et al (2018) Tumor-infiltrating lymphocytes in androgen receptor-positive, triple-negative breast cancer J Clin Oncol 36(5_suppl) 5-5 https://doi.org/10.1200/JCO.2018.36.5_suppl.5 
12. Castaneda C, Castillo M, and Enciso J, et al (2019) Role of undifferentiation markers and androgen receptor expression in triple-negative breast cancer Breast J 25(6) 1316-1319 https://doi.org/10.1111/tbj.13464 PMID: 31332870

13. Asano Y, Kashiwagi S, and Goto W, et al (2017) Expression and clinical significance of androgen receptor in triple-negative breast cancer Cancers 9(12) 4 https://doi.org/10.3390/cancers9010004 PMCID: 5295775

14. Jongen $\mathrm{L}$, Floris $\mathrm{G}$, and Wildiers $\mathrm{H}$, et al (2019) The prognostic role of the androgen receptor in patients with triple-negative early breast cancers and primary surgery J Clin Oncol 37(15_suppl) e12042-e12042 https://doi.org/10.1200/JCO.2019.37.15_suppl.e12042

15. Guiu S, Mollevi C, and Charon-Barra C, et al (2018) Prognostic value of androgen receptor and FOXA1 co-expression in non-metastatic triple negative breast cancer and correlation with other biomarkers Brit J Cancer 119(1) 76-79 https://doi.org/10.1038/s41416-0180142-6 PMID: 29880907 PMCID: 6035246

16. Traina T, Miller K, and Yardley D, et al (2015) Results from a phase 2 study of enzalutamide (ENZA), an androgen receptor (AR) inhibitor, in advanced AR+ triple-negative breast cancer (TNBC) J Clin Oncol 33(15_suppl) 1003-1003 https://doi.org/10.1200/jco.2015.33.15 suppl.1003

17. Dieci M, Tsvetkova V, and Griguolo G, et al (2019) Androgen receptor expression and association with distant disease-free survival in triple negative breast cancer: analysis of 263 patients treated with standard therapy for stage I-III disease Front Oncol 9452 https:// doi.org/10.3389/fonc.2019.00452 PMID: 31245286 PMCID: 6563384

18. Sutton L, Cao D, and Sarode V, et al (2012) Decreased androgen receptor expression is associated with distant metastases in patients with androgen receptor-expressing triple-negative breast carcinoma Am J Clin Pathol 138(4) 511-516 https://doi.org/10.1309/ AJCP8AVF8FDPTZLH PMID: 23010705

19. Hu R, Dawood S, and Holmes M, et al (2011) Androgen receptor expression and breast cancer survival in postmenopausal women Clin Cancer Res 17(7) 1867-1874 https://doi.org/10.1158/1078-0432.CCR-10-2021 PMID: 21325075 PMCID: 3076683

20. McGhan L, McCullough A, and Protheroe C, et al (2013) Androgen receptor-positive triple negative breast cancer: a unique breast cancer subtype Ann Surg Oncol 21(2) 361-367 https://doi.org/10.1245/s10434-013-3260-7 PMID: 24046116

21. Xu M, Yuan Y, and Yan P, et al (2020) Prognostic significance of androgen receptor expression in triple negative breast cancer: a systematic review and meta-analysis Clin Breast Cancer 20(4) e385-e396 https://doi.org/10.1016/j.clbc.2020.01.002 PMID: 32139270

22. Balkenhol MCA, Vreuls W, and Wauters CAP, et al (2020) Histological subtypes in triple negative breast cancer are associated with specific information on survival Ann Diagn Pathol 46151490 https://doi.org/10.1016/j.anndiagpath.2020.151490 PMID: 32179443

23. Loi S, Sirtaine N, and Piette F, et al (2013) Prognostic and predictive value of tumor-infiltrating lymphocytes in a phase III randomized adjuvant breast cancer trial in node-positive breast cancer comparing the addition of docetaxel to doxorubicin with doxorubicin-based chemotherapy: BIG 02-98 J Clin Oncol 31(7) 860-867 https://doi.org/10.1200/JCO.2011.41.0902 PMID: 23341518

24. Adams S, Gray R, and Demaria S, et al (2014) Prognostic value of tumor-infiltrating lymphocytes in triple-negative breast cancers from two phase III randomized adjuvant breast cancer trials: ECOG 2197 and ECOG $1199 \mathrm{~J}$ Clin Oncol 32(27) 2959-2966 https://doi. org/10.1200/JCO.2013.55.0491 PMID: 25071121 PMCID: 4162494

25. Pruneri G, Vingiani $A$, and Bagnardi $V$, et al (2016) Clinical validity of tumor-infiltrating lymphocytes analysis in patients with triplenegative breast cancer Ann Oncol 27(2) 249-256 https://doi.org/10.1093/annonc/mdv571

26. Loi S, Drubay D, and Adams S, et al (2019) Tumor-infiltrating lymphocytes and prognosis: a pooled individual patient analysis of earlystage triple-negative breast cancers J Clin Oncol 37(7) 559-569 https://doi.org/10.1200/JCO.18.01010 PMID: 30650045 PMCID: 7010425

27. Lotfinejad P, Asghari Jafarabadi M, and Abdoli Shadbad M, et al (2020) Prognostic role and clinical significance of tumor-infiltrating lymphocyte (TIL) and programmed death ligand 1 (PD-L1) expression in triple-negative breast cancer (TNBC): a systematic review and meta-analysis study Diagnostics 10(9) 704 https://doi.org/10.3390/diagnostics10090704 PMCID: 7554852 
28. Leon-Ferre RA, Polley MY, and Liu H, et al (2019) Characteristics, outcomes and prognostic factors of luminal androgen receptor (LAR) triple-negative breast cancer (TNBC) [abstract] Proceedings of the 2018 San Antonio Breast Cancer Symposium (Dec 2018) vol. 79 (Philadelphia: AACR) pp 3-8

29. Sánchez-Cousido L, López-González A, and Honrado Franco E, et al (2018) Relationship between androgen receptor and tumor-infiltrating lymphocytes in triple-negative breast cancer Ann Oncol 29 viii80 https://doi.org/10.1093/annonc/mdy270.246

30. Sánchez-Cousido L, Honrado Franco E, and Sanz Guadarrama Ó, et al (2020) 219P Relationship among PDL1, androgen receptor (AR) and tumour infiltrating lymphocytes (TILs) in early triple negative breast cancer Ann Oncol 31 S328-S329 https://doi.org/10.1016/j. annonc.2020.08.341

31. Liu Z, Li M, and Jiang Z, et al (2018) A comprehensive immunologic portrait of triple-negative breast cancer Translat Oncol 11(2) 311-329 https://doi.org/10.1016/j.tranon.2018.01.011

32. Mahmoud S, Paish E, and Powe D, et al (2011) Tumor-infiltrating CD8+ lymphocytes predict clinical outcome in breast cancer J Clin Oncol 29(15) 1949-1955 https://doi.org/10.1200/JCO.2010.30.5037 PMID: 21483002

33. Liu S, Lachapelle J, and Leung S, et al (2012) CD8+ lymphocyte infiltration is an independent favorable prognostic indicator in basal-like breast cancer Breast Cancer Res 14(2) R48 https://doi.org/10.1186/bcr3148 PMID: 22420471 PMCID: 3446382

34. Stanton S, Adams S, and Disis M (2016) Variation in the incidence and magnitude of tumor-infiltrating lymphocytes in breast cancer subtypes JAMA Oncol 2(10) 1354 https://doi.org/10.1001/jamaoncol.2016.1061 PMID: 27355489

35. Mahmoud S, Lee A, and Paish E, et al (2011) The prognostic significance of B lymphocytes in invasive carcinoma of the breast Breast Cancer Res Treat 132(2) 545-553 https://doi.org/10.1007/s10549-011-1620-1 PMID: 21671016

36. Mi H, Gong C, and Sulam J, et al (2020) Digital pathology analysis quantifies spatial heterogeneity of CD3, CD4, CD8, CD20, and FoxP3 immune markers in triple-negative breast cancer Front Physiol 11583333 https://doi.org/10.3389/fphys.2020.583333 PMID: 33192595 PMCID: 7604437

37. Keren L, Bosse M, and Marquez D, et al (2018) A structured tumor-immune microenvironment in triple negative breast cancer revealed by multiplexed ion beam imaging Cell 174(6) 1373-1387 https://doi.org/10.1016/j.cell.2018.08.039 PMID: 30193111 PMCID: 6132072

38. Goff S, and Danforth D (2021) The role of immune cells in breast tissue and immunotherapy for the treatment of breast cancer Clin Breast Cancer 21(1) e63-e73 https://doi.org/10.1016/j.clbc.2020.06.011

39. Elghazaly H, Rugo HS, and Azim HA, et al (2021) Breast-gynaecological \& immuno-oncology international cancer conference (BGICC) consensus and recommendations for the management of triple-negative breast cancer Cancers 13(9) 2262 https://doi.org/10.3390/ cancers13092262 


\section{Supplementary Material}

Table S1. Patients' characteristics of the whole cohort (36 patients).

\begin{tabular}{|c|c|c|c|}
\hline \multicolumn{2}{|c|}{ Patients' characteristics } & \multirow{2}{*}{$\begin{array}{c}\text { No }=36 \\
26 \\
10\end{array}$} & \multirow{2}{*}{$\begin{array}{c}\% \\
72.2 \\
27.8 \\
\end{array}$} \\
\hline Age category & $\begin{array}{l}\text { Age }<60 \text { years } \\
\text { Age } \geq 60 \text { years }\end{array}$ & & \\
\hline \multirow{2}{*}{ Menopausal status } & Pre-menopausal & 11 & 30.5 \\
\hline & Post-menopausal & 25 & 69.5 \\
\hline \multirow{4}{*}{ Laterality } & Bilateral & 2 & 5.6 \\
\hline & Right & 17 & 47.2 \\
\hline & Left & 16 & 44.4 \\
\hline & Unknown & 1 & 2.8 \\
\hline Pathology & $\begin{array}{l}\text { IDC } \\
\text { Medullary } \\
\text { carcinoma } \\
\text { ILC } \\
\text { Adenoid cystic } \\
\text { Unknown }\end{array}$ & $\begin{array}{c}21 \\
8 \\
5 \\
1 \\
1\end{array}$ & $\begin{array}{c}58.3 \\
22.2 \\
13.9 \\
2.8 \\
2.8\end{array}$ \\
\hline \multirow{3}{*}{ Grade } & Grade II & 11 & 30.6 \\
\hline & Grade III & 19 & 52.8 \\
\hline & Unknown & 6 & 16.6 \\
\hline \multirow{3}{*}{ Neo-adjuvant chemotherapy } & Negative & 31 & 86.1 \\
\hline & Positive & 3 & 8.3 \\
\hline & Unknown & 2 & 5.6 \\
\hline \multirow{2}{*}{ Type of surgery } & MRM & 30 & 83.3 \\
\hline & $\mathrm{BCS}$ & 6 & 16.7 \\
\hline \multirow{3}{*}{ Adjuvant chemotherapy } & Negative & 3 & 8.3 \\
\hline & Positive & 30 & 83.4 \\
\hline & Unknown & 3 & 8.3 \\
\hline \multirow{3}{*}{ Adjuvant radiotherapy } & Negative & 7 & 19.4 \\
\hline & Positive & 23 & 63.9 \\
\hline & Unknown & 6 & 16.7 \\
\hline TNM staging & $\begin{array}{l}\text { I } \\
\text { II } \\
\text { III } \\
\text { IV } \\
\text { Unknown }\end{array}$ & $\begin{array}{c}2 \\
11 \\
19 \\
3 \\
1 \\
\end{array}$ & $\begin{array}{c}5.6 \\
30.5 \\
52.8 \\
8.3 \\
2.8 \\
\end{array}$ \\
\hline T stage & $\begin{array}{l}\text { T1 } \\
\text { T2 } \\
\text { T3 } \\
\text { T4 } \\
\text { Unknown }\end{array}$ & $\begin{array}{c}5 \\
18 \\
7 \\
5 \\
1\end{array}$ & $\begin{array}{c}13.9 \\
50.0 \\
19.4 \\
13.9 \\
2.8\end{array}$ \\
\hline
\end{tabular}


Table S1. Patients' characteristics of the whole cohort (36 patients). (Continued)

\begin{tabular}{|l|l|c|c|}
\hline \multirow{5}{*}{ N stage } & No & 8 & 22.2 \\
& N1 & 9 & 25.0 \\
& N2 & 13 & 36.1 \\
& N3 & 5 & 13.9 \\
& Unknown & 1 & 2.8 \\
\hline \multirow{3}{*}{ LN category } & No & 8 & 22.2 \\
& N+ & 28 & 77.8 \\
\hline \multirow{3}{*}{ Relapse/progression } & Negative & 27 & 75.0 \\
\cline { 2 - 4 } & Positive & 9 & 25.0 \\
\cline { 2 - 4 } & Unknown & 0 & 0.0 \\
\hline \multirow{3}{*}{ Type of relapse (=9) } & Systemic & 6 & 66.7 \\
\cline { 2 - 4 } & Regional & 1 & 11.1 \\
\cline { 2 - 4 } & Unknown & 2 & 22.2 \\
\hline \multirow{3}{*}{ Systemic relapse (=6) } & Liver & 1 & 16.6 \\
& Brain & 2 & 16.6 \\
& Lung, bone & 2 & 33.4 \\
\hline 3-year OS & Unknown & 16 & 33.4 \\
\hline & & & 44.4 \\
\hline
\end{tabular}

IDC, Invasive ductal carcinoma; ILC, Invasive lobular carcinoma; MRM, Modified radical mastectomy; BCS, Breast conservative surgery; LN, Lymph node; OS, Overall survival

Table S2. Relation between age and the total and differential TILs.

\begin{tabular}{|c|c|c|c|c|c|}
\hline \multirow{2}{*}{\multicolumn{2}{|c|}{ Total and differential TILs }} & \multicolumn{2}{|c|}{ Age } & \multirow{2}{*}{ Test value } & \multirow{2}{*}{$p$-value } \\
\hline & & \multirow{2}{*}{$\begin{array}{c}\text { Age }<60 \\
38(10-50)\end{array}$} & \multirow{2}{*}{$\begin{array}{l}\text { Age } \geq 60 \\
10(3-50)\end{array}$} & & \\
\hline \multirow{2}{*}{ Total TILs (\%) } & Median (IQR) & & & \multirow{2}{*}{$-0.745^{b}$} & \multirow{2}{*}{0.456} \\
\hline & Range & $1-60$ & $1-70$ & & \\
\hline \multirow{2}{*}{ Median CD20 (\%) } & Median (IQR) & $18(10-20)$ & $10(10-20)$ & \multirow{2}{*}{$-0.156^{b}$} & \multirow{2}{*}{0.876} \\
\hline & Range & $0-40$ & $1-35$ & & \\
\hline \multirow{2}{*}{ Mean CD3 (\%) } & Mean \pm SD & $83.64 \pm 10.14$ & $73.00 \pm 28.54$ & \multirow{2}{*}{$1.554^{\mathrm{a}}$} & \multirow{2}{*}{0.131} \\
\hline & Range & $60-100$ & $2-95$ & & \\
\hline \multirow{2}{*}{ Median CD4 (\%) } & Median (IQR) & $5(0-20)$ & $0(0-10)$ & \multirow{2}{*}{$-0.933^{b}$} & \multirow{2}{*}{0.351} \\
\hline & Range & $0-30$ & $0-20$ & & \\
\hline \multirow{2}{*}{ Mean CD8 (\%) } & Mean \pm SD & $76.36 \pm 14.24$ & $66.33 \pm 29.00$ & \multirow{2}{*}{$1.302^{\mathrm{a}}$} & \multirow{2}{*}{0.203} \\
\hline & Range & $50-100$ & $2-90$ & & \\
\hline
\end{tabular}

IQR, Interquartile range; SD, Standard deviation

andependent $t$-test

bMann-Whitney test 
Table S3. Relation between age and the total and differential TILs.

\begin{tabular}{|c|c|c|c|c|c|c|c|}
\hline \multirow{3}{*}{\multicolumn{2}{|c|}{ Total and differential TILs }} & \multicolumn{4}{|c|}{ Age } & \multirow{3}{*}{ Chi-square test } & \multirow{3}{*}{$p$-value } \\
\hline & & \multicolumn{2}{|c|}{ Age $<60$} & \multicolumn{2}{|c|}{ Age $\geq 60$} & & \\
\hline & & No. & $\%$ & No. & $\%$ & & \\
\hline \multirow{2}{*}{ LDBC versus $\mathrm{LPBC}$} & LDBC & 15 & 68.2 & 6 & 66.7 & \multirow{2}{*}{0.007} & \multirow{2}{*}{0.935} \\
\hline & LPBC & 7 & 31.8 & 3 & 33.3 & & \\
\hline \multirow{2}{*}{ CD20 expression } & Negative & 3 & 13.6 & 0 & 0.0 & \multirow{2}{*}{1.359} & \multirow{2}{*}{1.000} \\
\hline & Positive & 19 & 86.4 & 9 & 100 & & \\
\hline \multirow{2}{*}{ CD3 expression } & Negative & 0 & 0.0 & 0 & 0.0 & \multirow{2}{*}{ NA } & \multirow{2}{*}{ NA } \\
\hline & Positive & 22 & 100.0 & 9 & 100 & & \\
\hline \multirow{2}{*}{ CD4 expression } & Negative & 11 & 50.0 & 6 & 66.7 & \multirow{2}{*}{0.716} & \multirow{2}{*}{0.397} \\
\hline & Positive & 11 & 50.0 & 3 & 33.3 & & \\
\hline \multirow{2}{*}{ CD8 expression } & Negative & 0 & 0.0 & 0 & 0.0 & \multirow{2}{*}{ NA } & \multirow{2}{*}{ NA } \\
\hline & Positive & 22 & 100.0 & 9 & 100 & & \\
\hline
\end{tabular}

N, Not applicable

Table S4. Correlation between the total and differential TILs expression. The $p$-value was considered significant if $\leq 0.05$.

\begin{tabular}{|c|c|c|c|c|c|c|c|c|c|c|}
\hline & \multicolumn{2}{|c|}{ Total TILs (\%) } & \multicolumn{2}{|c|}{ CD20 (\%) } & \multicolumn{2}{|c|}{ CD3 (\%) } & \multicolumn{2}{|c|}{ CD4 (\%) } & \multicolumn{2}{|c|}{ CD8 (\%) } \\
\hline & $r$ & $p$-value & $r$ & $p$-value & $r$ & $p$-value & $r$ & $p$-value & $r$ & $p$-value \\
\hline Total TILs (\%) & - & - & 0.228 & 0.218 & -0.151 & 0.416 & -0.008 & 0.964 & -0.060 & 0.747 \\
\hline CD20 (\%) & 0.228 & 0.218 & - & - & -0.814 & $<0.001$ & 0.200 & 0.282 & -0.382 & 0.034 \\
\hline CD3 (\%) & -0.151 & 0.416 & -0.814 & $<0.001$ & - & - & -0.101 & 0.590 & 0.591 & $<0.001$ \\
\hline CD4 (\%) & -0.008 & 0.964 & 0.200 & 0.282 & -0.101 & 0.590 & - & - & -0.527 & 0.002 \\
\hline CD8 (\%) & -0.060 & 0.747 & -0.382 & 0.034 & 0.591 & $<0.001$ & -0.527 & 0.002 & - & - \\
\hline
\end{tabular}

